\title{
Effects of stimulus intensity on the habituation of flexor withdrawal activity mediated by the functionally transected human spinal cord
}

\author{
MARCUS J. FUHRER \\ Departments of Rehabilitation \& Psychiatry, Baylor College of Medicine \\ and \\ Texas Institute for Rehabilitation and Research, Houston, Texas 77030
}

\begin{abstract}
Ten patients with a chronic, transverse lesion of the cervical spinal cord were submitted to two daily sessions involving a relatively high- or low-intensity habituating stimulus applied unilaterally to a midplantar site at the rate of $1 / \mathrm{sec}$. Test stimuli of relatively high, intermediate, and low intensity were applied before and after habituation training. Response measures were based upon the integrated electromyographic activity of the tibialis anterior muscle. Analyses of both absolute and relative responses measures obtained during and at the termination of habituation training indicated that the degree of habituation was inversely related to the intensity of stimulation. The incremental growth in responsiveness observed during the first few repetitions of the habituating stimuli was greater for strong stimulation than for weak. Analyses of responses to test stimuli applied before and after habituating training suggested that the effects of the strong habituating stimulus generalized more to a posthabituation test stimulus of intermediate intensity than did the effects of the weak habituating stimulus.
\end{abstract}

It is now well established in both intact and spinal mammalian preparations that withdrawal reflexes exhibit the form of plasticity known as habituation, i.e., a decrement in responsiveness to repetitive afferent stimulation that is not attributable to either sensory adaptation or effector fatigue. In addition to research on neural mechanisms involved in the habituation of withdrawal activity (Groves \& Thompson, 1973), substantial investigative effort has been devoted to delineating parameters of the stimulus conditions that influence the process. Chief among these parameters are the number, rate, and strength of the habituating stimuli. With respect to stimulus intensity, the focus of the present investigation, an influential review published approximately a decade ago contained the empirical generalization that "the weaker the stimulus, the more rapid and/or more pronounced is habituation. Strong stimuli may yield no significant habituation" (Thompson \& Spencer, 1966, p. 19). The subsequent research, critical discussions, and theorization suggest instead that the effects of stimulus intensity may vary considerably depending upon the experi-

The skilled technical assistance of Mr. Robert Townsend and Ms. Gayle Birk is gratefully acknowledged. This work was conducted at the Texas Institute for Rehabilitation and Research, and was supported by Research Grant NS 07755-08 from the National Institute of Neurological and Communicative Disorders and Stroke and by Rehabilitation Services Administration Grant 16-P-56813-6/14 to Rehabilitation Research and Training Center No. 4. mental arrangements and response measures that are chosen.

Thompson, Groves, Teyler, and Roemer (1973) have pointed out how outcomes may differ prominently, depending upon whether absolute or relative measures of habituation are used. Davis and Wagner (1968) have identified what they view as a fundamental flaw in the experimental design used in most studies of stimulus intensity effects upon habituation. They point out that the usual procedural arrangement confounds differences in the stimuli used to achieve habituation with differences in the intensity of stimuli used to test the effects of habituation training. A remedy is proposed in which test stimuli of intensities other than those used in the habituating series are administered before and after habituation training. These and other relevant caveats were taken into consideration in the design of the present study concerned with the effects of different stimulus intensities upon habituation of the lower extremity withdrawal reflex mediated by the cervically transected human spinal cord.

\section{METHOD}

The 10 male subjects were between 18 and 34 years of age. Each had sustained a traumatic injury of the cervical spinal cord between the fourth and seventh segments and, at the time of the study, were 15-83 months postinjury. Each individual was examined neurologically by at least two examiners prior to admission into the study, and in no case was voluntary motor functioning or sensation detected below the level of the lesion. A test (Fuhrer, 1971b) also was conducted to demonstrate that electrodermal 
activity of the volar surfaces was independent of suprasegmental control. The subjects all had been discharged from a comprehensive rehabilitation program and were, with one exception, either employed or attending a university. They were all free of decubitus ulcers or clinical signs of urinary tract infection, and were withdrawn from all spasmolytic medications no less than $48 \mathrm{~h}$ preceding each study session.

The oscillograph, instrumentation used to record the EMG, special-purpose skin conductance monitor, stimulator, and timing equipment have been described previously (Fuhrer, 1971a).

Each person participated in two daily sessions that were conducted, to the extent possible, on consecutive days at the same hour. The temperature in the laboratory was maintained between $22^{\circ}$ and $24^{\circ} \mathrm{C}$. With the individual in a supine position on an examination table, percussion was applied to the suprapubic area in order to empty the bladders of the five subjects who did not have an indwelling urethral catheter. Clip-on electrodes (Dimitrijevic \& Nathan, 1970) for applying electrocutaneous stimulation were affixed to the medial plantar aspect of the right foot. The silver-silver chloride electrodes for recording EMG activity were placed over the tibialis anterior and rectus femoris muscles of the right leg, and the electrogonimeter was attached to the right ankle.

A work-up procedure then was begun to establish the intensity of stimulation to be used. The constant-current stimulator was adjusted to supply a $40-\mathrm{msec}$ pulse train at the frequency of $100 \mathrm{~Hz}$ and a pulse duration of $1 \mathrm{msec}$. The current level was set initially at $0.2 \mathrm{~mA}$ and was increased in $0.2-\mathrm{mA}$ steps at $30-\mathrm{sec}$ intervals until a just-observable EMG response was recorded three times in succession from the tibialis anterior muscle. This current level was defined as threshold. Three different stimulus intensities then were defined: a weak intensity that was twice the threshold current, a strong intensity that was five times the threshold current, and an intermediate intensity that was three and one-half times the threshold current. Following a 3-min stimulus-free interval, prehabituation responsiveness was assessed by applying three repetitions each of the strong-, intermediate-, and weak-intensity stimuli in a counterbalanced order with an interstimulus interval of $10 \mathrm{sec}$. Habituation training then began; this comprised 100 repetitions of either the weak- or strong-intensity stimulus at the rate of $1 / \mathrm{sec}$. The posthabituation series of test stimuli consisted of three repetitions each of the strong, intermediate, and weak intensity stimuli in a counterbalanced order with an interstimulus interval of $10 \mathrm{sec}$. The decision of whether a person's first session would involve the strong or weak habituating stimulus was decided by a coin toss. Throughout the period of repetitive stimulation, the subject was requested to inhibit conversation and voluntary movements to minimize effects on the excitability of spinal reflexes.

Quantification of the EMG responses was achieved by circuitry which rectified and integrated EMG activity over a 900 -msec interval beginning $50 \mathrm{msec}$ after stimulus onset. Approximately 2 msec thereafter, an oscillographically recorded pulse of 380 -msec duration was produced with an amplitude proportional to the integrated value. The pulse, an event marker, and time code also were fed into separate channels of an FM tape recorder (CEC Model VR-3300) to permit subsequent computer processing of the integrated EMG data. The electrogoniometer used to record excursions of the ankle joint yielded a 3-mm, oscillographically recorded deflection for each degree of joint excursion.

\section{RESULTS}

It was observed visually that within the range of stimulus intensities used, lower extremity movements were limited almost exclusively to the limb being stimulated. The principal differences between the effects of strong and weak stimulation consisted of the number of joints involved in the withdrawal movement as well as their amplitude and briskness. The pattern of responses to the stimulus of weak intensity presented prior to habituation training was limited characteristically to dorsiflexion of the toes and ankle. Following the inception of $1 / \mathrm{sec}$ stimulation, the flexion pattern took on a rhythmic character and became more widespread to include not only more vigorous ankle flexion but also a degree of knee and hip flexion. As repetitive stimulation continued, the latter response components dropped out altogether and the ankle and toe movements diminished progressively in amplitude. By the 100th repetition of $1 / \mathrm{sec}$ stimulation, visible movement either was totally extinguished or limited to rhythmic, slight dorsiflexion of the ankle and hallux. The response pattern to strong intensity stimulation presented prior to habituation training consisted typically of brisk dorsiflexion of the toes and ankle acompanied by moderate knee and hip flexion. Flexion movements at all these joints become larger and more vigorous during the first few repetitions of the stimulus at the rate of $1 / \mathrm{sec}$. As stimulation continued, however, the movements decreased in amplitude with the knee and hip excursions being the first to drop out altogether. All subjects continued to exhibit some degree of rhythmic ankle and toe movements at the termination of strong intensity, $1 /$ sec stimulation.

Two response measures were analyzed quantitatively, one being integrated EMG activity from the tibialis anterior muscle, the other being the goniometrically recorded position of the ankle joint. Rectus femoris EMG activity was not analyzed because it was absent in the recordings of five subjects when test presentations of weak stimulation were applied. Separate correlational analyses based upon each subject's data (in microvolt-seconds corrected for differences in amplifier gain) indicated that the integrated responses from the tibialis anterior muscle and the goniometric measures of ankle flexion were virtually redundant in amplitude, the median correlation coefficient being .94 . Consequently, only the integrated EMG data are reported. It should be noted that the integrated EMG response to each stimulus was corrected by subtracting from it a value reflecting background activity integrated over an identical period of time.

The data points contained in Figure 1 represent the median amplitude of integrated EMG activity (in microvolt seconds) to strong and weak habituating stimuli. The values labeled "PRE" are based upon each subject's amplitude of responses to the three initial test presentations of the stimulus. The succeeding median values represent the average amplitude of responses to three adjacent trials during habituation training with $1 / \mathrm{sec}$ stimulation. As reflected by the values for Trials $2-4$, response ampli- 


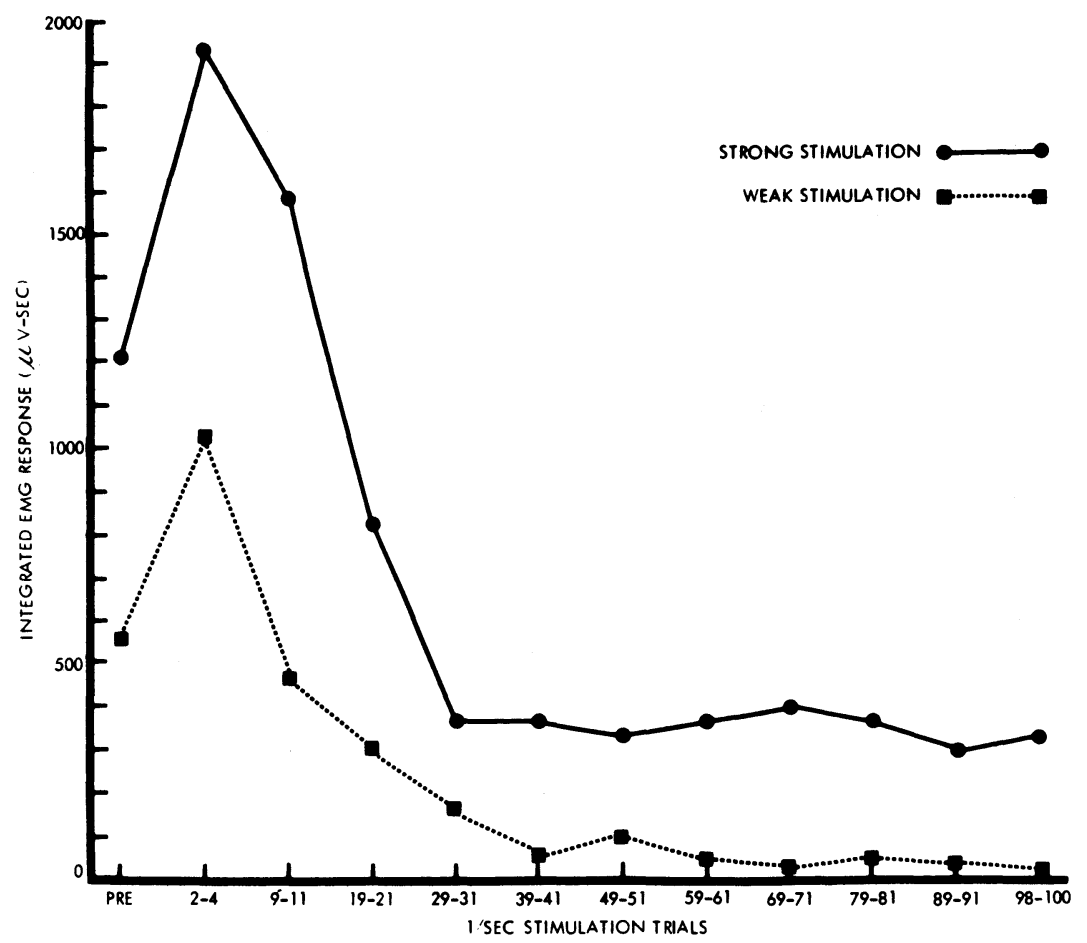

Figure 1. Median amplitude of integrated EMG activity (in microvolt-seconds) to strong and weak habituating stimuli.

tudes initially rose substantially above baseline levels when either strong or weak intensity stimuli were applied. This increased responsiveness was exhibited by all subjects in both conditions. The indication that strong stimulation was associated with a larger increase in responsiveness than weak stimulation was assessed statistically by subtracting each subject's value for "PRE" from the values for Trials 2-4 and 9-11. For both sets of difference scores, $\mathrm{t}$ tests confirmed that the increase in responding during strong stimulation was reliably greater than that observed during weak stimulation $(p<.05)$. It may be noted in Figure 1 that almost all of the decline in response amplitudes occurred between Trials 9-11 and 39-41. Throughout the remaining trials, the response levels to strong stimulation remained consistently and reliably higher than to weak stimulation $(\mathrm{p}<.01)$.

The data pertaining to the median relative response magnitudes are displayed in Figure 2 . The values for individual subjects were obtained by dividing the average amplitude of responses for each triad of trials by the average amplitude of responses to the three initial test presentations of the stimulus. The median values for responses to strong and weak habituating stimuli during Trials $2-4$ did not differ significantly $(p>.10)$, but did during Trials $9-11$ $(p<.05)$. For all trials following Trials 29-31, responding was reliably greater to strong stimulation than to weak $(p<.05)$.
In Figure 3 are depicted the findings obtained by presenting test stimuli of strong, intermediate, and weak intensity prior to and following $1 / \mathrm{sec}$ habituation training with either strong or weak intensity stimulation. The data for each subject were based upon the average amplitude of responses to three repetitions of the stimulus at each of the three intensities. The panel on the left depicts the results in terms of median absolute values, while the panel on the right displays the median relative values obtained by expressing responses to posthabituation test trials as a percentage of responses to prehabituation test trials. Scrutiny of the posthabituation values reveals that responding to the test stimuli of strong and intermediate intensities was more depressed following habituation training with strong stimulation than with weak. These findings were statistically significant for both the absolute and relative response measures $(p<.05)$. The amplitude of responses to posthabituation test stimuli of weak intensity did not differ significantly as a function of using a weak or strong habituating stimulus.

\section{DISCUSSION}

The present results indicate that differences in stimulus intensity indeed have a demonstrable effect upon-habituation of the lower extremity withdrawal response mediated by the functionally 


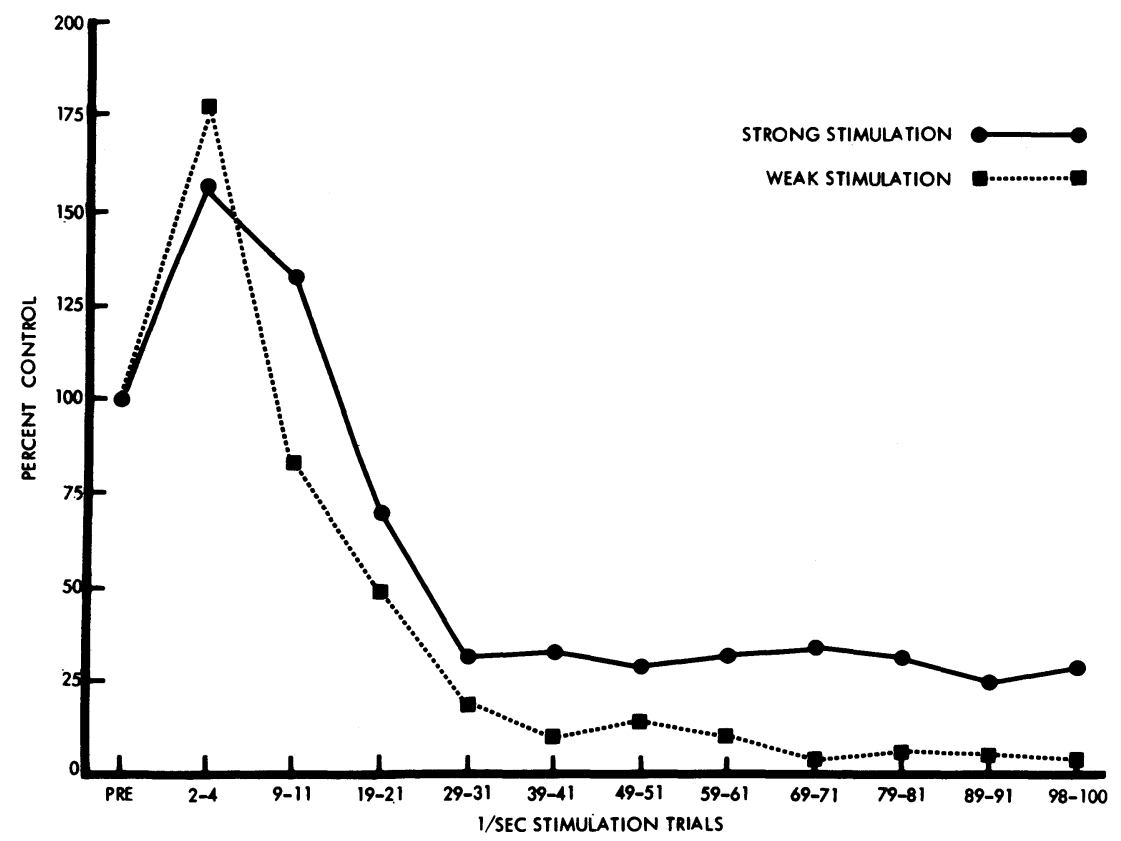

Figure 2. Median percentage response to strong and weak habituating stimuli, based upon control level responsiveness.

transected human spinal cord. It is clear also that outcomes are influenced by the arrangement of test stimuli and, to a lesser extent by the response measures that are used.

When the effects of strong and weak habituating stimuli were assessed during habituation training and at its termination, response levels were lower in association with a relatively weak habituating stimulus than with a strong one. This result was obtained using both absolute and relative response measures.
The indication that habituation is inversely related to the strength of the habituating stimulus is consistent with results obtained for the hindlimb flexion reflex of the acute spinal cat (Groves, Lee, \& Thompson, 1969; Thompson \& Spencer, 1966). The present findings also substantiate previously reported, illustrative data for spinal man, suggesting that the amplitude of flexor EMG activity from the lower extremity declines more rapidly with relatively weak habituating stimuli (Dimitrijevic \& Nathan,
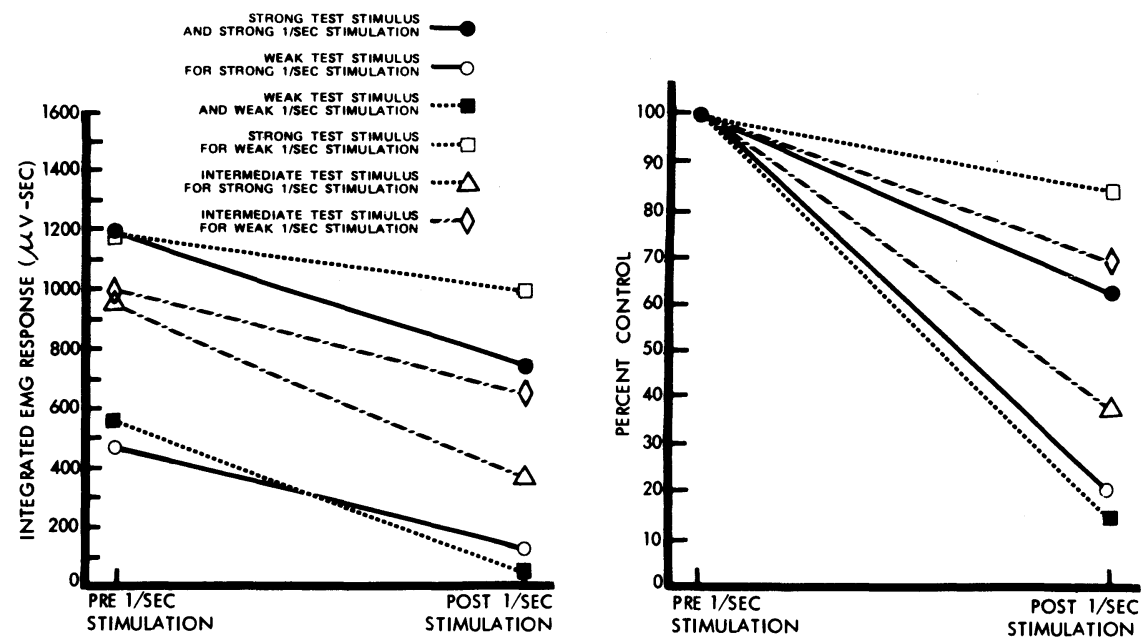

Figure 3. Median responsiveness to test stimuli of strong, intermediate, and weak intensity prior to and following $1 / \mathrm{sec}$ habituation training with either strong- or weak-intensity stimuli. The data for the absolute response measure (integrated EMG activity in microvolt-seconds) are shown in the panel on the left; the data for the relative response measure (percentage response based upon control level responsiveness) are shown in the right panel. 
1970). In contrast to the findings that have been cited, the responsiveness of the flexor reflex of the spinal rat at the termination of a series of habituating stimuli has been reported to be greater in conjunction with a weak habituating stimulus than with a strong one (Pearson \& MacDonald, 1973). Among the factors that may have contributed to these exceptional results are use of an atypically lengthy $(10 \mathrm{sec})$ interval among habituating stimuli and the unconventional response measure which was based upon integrating a relatively late component of the EMG afterdischarge $500 \mathrm{msec}$ following onset of the stimulating pulse train.

It was observed consistently that the pattern of sequential changes in response amplitudes to repetitive stimulation of the plantar surface was not solely decremental in character; rather, the amplitude of responses increased during the first few stimuli in the series. A similar initial increase in lower extremity withdrawal activity was observed in previous studies using stimulus repetition rates of $1 / \mathrm{sec}$ (Fuhrer, 1976) or $2 / \mathrm{sec}$ (Fuhrer, 1973). This incremental pattern of responding is generally termed "sensitization" (Groves et al., 1969) and is attributed to a process separate from the one underlying habituation (Groves \& Thompson, 1970). Consistent with evidence obtained for the flexor withdrawal reflex of both the spinal cat and rat (Pearson \& Richardson, 1975), sensitization has been postulated to be related directly to the intensity of stimulation (Groves \& Thompson, 1970). This hypothesis is generally supported by the present results, e.g., analysis of the absolute response measure indicated that the initial increment in responsiveness was greater with the strong habituating stimulus than with the weak one. Using a relative response measure which to some extent factors out differences in response strength existing prior to repetitive stimulation, no difference was found in the peak response amplitude that was reached, but the increase in responsiveness over prehabituation levels was found to be sustained longer during habituation training with the strong stimulus.

According to a view developed by Davis and Wagner (1968), a serious logical flaw pervades the kind of analyses that have been discussed. They point out that the comparisons confound the intensities of the habituating and test stimuli, i.e., that the observed differences in responding may be ascribed logically not only to differences in the intensity of the preceding series of stimuli used for habituation training, but also to differences in the intensity of stimuli comprising test trials, or to both factors. A remedial experimental design is recommended in which a stimulus intensity other than the strong or weak one used for habituation training is administered to test the effects of each. Based upon this design, it was found that habituation of the rat's startle response to an auditory stimulus was directly related to the intensity of the stimulus. Using the same essential design in a study of motoneurons in the acute spinal cat, Wickelgren (1967) also observed that habituation increased with the intensity of the habituating stimulus. Results suggesting the opposite relation between habituation and stimulus intensity were obtained in a later study (Groves, Lee, \& Thompson, 1969) in which flexor muscle contractions of the acute spinal cat were recorded in connection with procedures that purportedly replicated those of Wickelgren (1967). However, the description of the experimental procedures suggests that the key provision of using test stimuli of intensities other than those involved in habituation training was not implemented. In the present study, this was achieved by using a test stimulus intensity intermediate to the levels used for the weak and strong habituating stimuli. Comparisons of the amplitude of responses to the intermediate test stimulus before and after habituation training revealed reliably lower response amplitudes following habituation with a strong stimulus than following habituation with a weak one. This was true regardless of whether an absolute or relative response measure was used.

This discussion would be incomplete without noting that Thompson et al. (1973) have contested the kind of experimental design recommended by Davis and Wagner (1968). It is argued that the necessary and sufficient condition for the occurrence of habituation is repetition of the stimulus. Altering the intensity of stimulation between habituation training and testing is noted as being beyond the critical defining condition. It is suggested further that the latter operation actually defines the distinctly different phenomenon of "generalization along the dimension of stimulus intensity." The extent of generalization from the habituating stimulus to test stimuli of other intensities is viewed as depending upon the degree of overlap between the interneuronal networks that are involved. From this standpoint, the present results suggest that the effects of a weak habituating stimulus generalize less to a posthabituating test stimulus of intermediate intensity than do the effects of a strong habituating stimulus. This outcome is predicted explicitly by Thompson et al. (1973) on the ground that a smaller percentage of interneurons mediating the response to the intermediate intensity test stimulus was activated and thus habituated by the weak habituating stimulus then by the strong one. In the same discussion, a number of highly specific predictions are derived from the "dual process" theory of habituation which are potentially relevant to the present investigation. These predictions, however, are qualified by a number of stipulations, one being that the stimulus 
conditions result in response habituation that is complicated little, if any, by sensitization. As has been noted, however, this was not the case in the present study regardless of whether a strong or a weak habituating stimulus was used. Another qualification is that responses to test stimuli presented following habituation training do not reflect spontaneous recovery. Comparison of the terminal response levels depicted in Figures 1 and 2 with data for the posthabituation test trials shown in Figure 3 make it clear that a degree of spontaneous recovery did occur in the present study, especially in response to the strong stimulus.

\section{REFERENCES}

DAvis, M., \& WAGNER, A. R. Startle responsiveness after habituation to different intensities of tone. Psychonomic Science, $1968,12,337-338$.

Dimitrijevic, M. R., \& Nathan, P. W. Studies of spasticity in man. 4. Changes in flexion reflex with repetitive cutaneous stimulation in spinal man. Brain, 1970, 93, 743-768.

FUHRER, M. J. Skin conductance responses mediated by the transected human spinal cord. Journal of Applied Physiology, 1971, 30, 663-669. (a)

FUhrer, M. J. Analysis of electrodermal evidence for a paramedullary afferent tract in patients with a transection of the thoracic spinal cord. Journal of Neurology, Neurosurgery, \& Psychiatry, 1971, 34, 281-288. (b)

FUHRER, M. J. Dishabituation of flexor withdrawal activity mediated by the functionally transected human spinal cord. Brain Research, 1973, 63, 93-102.
FUHRER, M. J. Interstimulus interval effects on habituation of flexor withdrawal activity mediated by the functionally transected human spinal cord. Archives of Physical Medicine \& Rehabilitation, 1976, 57, 577-582.

Groves, P. M., LeE, D., \& Thompson, R. F. Effects of stimulus frequency and intensity on habituation and sensitization in acute spinal cats. Physiology and Behavior, 1969, 4, 383-388.

Groves, P. M., \& Thompson, R. F. Habituation: A dual-process theory. Psychological Review, 1970, 77, 419-450.

Groves, P. M., \& Thompson, R. F. A dual-process theory of habituation: Neural mechanisms. In H. V. S. Peeke \& M. J. Herz (Eds.), Habituation: Physiological substrates (Vol. 2). New York: Academic Press, 1973. Pp. 175-206.

Pearson, J. A., \& MacDonald, J. R. Habituation and sensitization of the flexor reflex after asphyxiation of the spinal cord. Brain Research, 1973, 58, 427-435.

Pearson, J. A., \& Richardson, T. L. The influence of stimulus intensity on sensitization of the flexor reflex. Experimental Neurology, 1975, 47, 193-197.

Thompson, R. F., Groves, P. M., Teyler, T. J., \& Roemer, R. A. A dual-process theory of habituation: Theory and behavior. In H. V. S. Peeke \& M. J. Herz (Eds.), Habituation: Behavioral studies (Vol. 1). New York: Academic Press: 1973. Pp. 239-272.

Thompson, R. F., \& Spencer, W. A. Habituation: A model phenomenon for the study of neuronal substrates of behavior. Psychological Review, 1966, 73, 16-43.

WICKELGREN, B. G. Habituation of spinal mononeurons. Journal of Neurophysiology, 1967, 30, 1404-1423.

(Received for publication February 18, 1977; revision accepted April 26, 1977.) 